\title{
A Strategy for the Progressive Introduction of Picture Archiving and Communication Systems for a Radiology Department
}

\author{
Arthur F. Naylor
}

\begin{abstract}
An example of strategy for the progressive introduction of picture archiving communication systems (PACS) into a complete global image management system for a Department of Radiology has been developed and quantitatively examined. In general the strategy is based on the earliest reasonable implementation consistent with the evolution of the usage patterns and the state of the technology and its economics. The progressive implementation schedule and the associated numerical estimates of memory requirements in this paper are based on a series of qualitative and quantitative assumptions. The development of the projected strategy and schedule suggests that a time period of $1 \frac{1}{2}$ to 2 decades would be required for an aggressive institution to achieve an operational global PACS system.
\end{abstract}

KEY WORDS: image management system, PACS, implementation schedule, digital archive, memory requirement.

$\mathbf{T}$

HE STRATEGY and schedule for the progressive implementation of a global picture archiving and communication system (PACS) for a Department of Radiology are designed to be consistent with the early introduction of PACS for the inherently digital modalities and to allow for the extension to a succession of other radiological modalities and subspecialties as diagnostic benefits can be realized. They are also consistent with the expected evolution of the various computerrelated component technologies, the growth in software and systems capabilities, the evolution of sensitized plate imaging technology, and especially the evolution of the professional and institutional usage patterns. The schedule example is necessarily hypothetical as no institution has yet achieved the use of full digital technology to replace film-based archival and retrieval systems for all subspecialties and modalities of

From the A.F. Naylor Co, Cleveland, $\mathrm{OH}$.

No reprints available.

Adapted with permission from the following sources: Naylor A: Strategies for the progressive introduction of $P A C S$, in Lehmke HU, Rhodes ML, Jaffe CC, et al: Computer Assisted Radiology: Proceedings of the International Symposium. New York, NY, Springer-Verlag, 1991; Naylor A: Strategies for the progressive introduction of PACS. Radiol Diagn 33:22-27, 1992 radiology. The strategy for the introduction of PACS must concern both qualitative and quantitative considerations, because the implementation plan determines the magnitude of the required digital data environment and is determined by the growing magnitude and sophistication for which the digital systems can be reasonably implemented.

\section{STRATEGY AND SCHEDULE DEVELOPMENT}

Previous work ${ }^{1}$ has developed an outline of the status and expected factors in the evolution of the digital environment for radiology as a set of three contributing concurrent and interacting evolutions. These are the evolution of digital component technologies, systems configurations, and professional and institutional usage patterns.

Consideration of these factors has led to the selection of a set of qualitative assumptions, which have been purposely chosen to be quite favorable, to influence the development of the projected schedule. These qualitative assumptions include the following factors:

1. Two highly qualified in-house PACS experts with exceptional interpersonal skills available full time for the program.

2. All radiologists fully cooperative with the PACS concept and with soft copy reading.

3. A fully cooperative administration, providing provision for adequate funds for all interim, permanent, supplementary and replacement equipment and software; adequate technical, administrative and support staff; adequate space for mainframe and peripheral equipment; and adequate dedicated communication raceways.

4. Eager cooperation for interactive improvements from PACS suppliers.

5. Cooperation from all acquisition equipment manufacturers regarding interfaces.

6. Cooperation from radiology information systems (RIS) and hospital information service (HIS) suppliers regarding interfaces and special requirements.

The anticipated environment is optimistic with regard to the assumed cooperation of the 
Table 1. Digital Format for the Example

\begin{tabular}{|c|c|c|c|c|c|c|}
\hline \multirow[b]{2}{*}{ Examination Type } & \multirow{2}{*}{$\begin{array}{l}\text { Matrix } \\
\text { Pixels }\end{array}$} & \multirow{2}{*}{$\begin{array}{c}\text { Mbytes/ } \\
\text { image }\end{array}$} & \multicolumn{2}{|c|}{ Images/Examination } & \multicolumn{2}{|c|}{ Mbytes/Examination } \\
\hline & & & Generated & Archived & Generated & Archived \\
\hline Diagnostic fluoroscopy & $2048^{2}$ & 8.000 & 8 & 8 & 64.0 & 64.0 \\
\hline Diagnostic radiography & $2048^{2}$ & 8.000 & 2.1 & 2.1 & 16.8 & 16.8 \\
\hline Mammography & $2048^{2}$ & 8.000 & 6 & 6 & 48.0 & 48.0 \\
\hline Angiography & $2048^{2}$ & 8.000 & 40 & 40 & 320.0 & 320.0 \\
\hline Portables & $2048^{2}$ & 8.000 & 1 & 1 & 8.0 & 8.0 \\
\hline Digital angiography & $1580^{2}$ & 5.000 & 46 & 46 & 230.0 & 230.0 \\
\hline Ultrasound & $512^{2}$ & 0.262 & 40 & 40 & 10.5 & 10.5 \\
\hline Nuclear medicine & $256^{2}$ & 0.131 & 6 & 6 & 0.8 & 0.8 \\
\hline Computed tomography & $512^{2}$ & 0.524 & 25 & 25 & 13.1 & 13.1 \\
\hline Magnetic resonance & $512^{2}$ & 0.524 & 28 & 28 & 14.6 & 14.6 \\
\hline
\end{tabular}

professional staff, hospital administration, and equipment suppliers and manufacturers as well as to unrestricted financial, space, and staffing resources.

The quantitative development of the plan is based on an extensive model of image generation factors, typical patient repeat visit patterns, film file dynamics, and the frequency and amount of image retrieval. The assumed digital content of each image and each examination for 10 different modalities and subspecialties of radiology is shown in Table 1 . The quantitative digital environment activity model has been documented in considerable detail. ${ }^{2}$ The amount of detailed information required in the appropriate digital image format for radiographic studies has been the subject of much discussion and the range of values that have been discussed are noted. ${ }^{1}$ There appears to be a growing consen- sus that about 8 Mbytes per image would provide appropriate image information for radiographic studies. While the spatial resolution would be greater with the smaller film sizes, such as those used for mammography and skull radiography, some believe that a somewhat greater information content might be required for mammography. The modeling for digital angiography has been changed slightly from that used in Reference 2. The assumed caseload distribution and the digital data distribution per 100,000 total annual examinations are shown in Table 2.

The numerical baseline for the model should be considered as illustrative of the methodology. Many of the factors, such as distribution of examinations by type, number of images per examination, and file retention period would normally be customized to make the model

Table 2. Activity Basis for the Example

\begin{tabular}{|c|c|c|c|c|c|c|c|}
\hline \multirow[b]{3}{*}{ Examination Type } & \multicolumn{4}{|c|}{ Yearly Data } & \multicolumn{3}{|c|}{ Typical Day } \\
\hline & \multirow[b]{2}{*}{ Exams } & \multirow{2}{*}{$\begin{array}{l}\text { Images } \\
(\times 1,000)\end{array}$} & \multirow{2}{*}{$\begin{array}{l}\text { Archive } \\
\text { (Gbytes) }\end{array}$} & \multirow{2}{*}{$\begin{array}{c}\text { Compressed } \\
\text { 1-yr Arch } \\
\text { (Gbytes) }\end{array}$} & \multirow[b]{2}{*}{ Exams } & \multicolumn{2}{|c|}{ Generated } \\
\hline & & & & & & Images & Mbytes \\
\hline Diagnostic fluoroscopy & 5,000 & 40.0 & 320.000 & 103.226 & 25.0 & 200 & 1,600 \\
\hline Diagnostic radiography & 45,000 & 94.5 & 756.000 & 243.871 & 225.0 & 473 & 3,780 \\
\hline Mammography & 3,000 & 18.0 & 144.000 & 46.452 & 15.0 & 90 & 720 \\
\hline Angiography & 1,000 & 40.0 & 320.000 & 103.226 & 5.0 & 200 & 1,600 \\
\hline Subtotal & 54,000 & 192.5 & 1540.000 & 496.774 & 270.0 & 963 & 7,700 \\
\hline Portables & 24,000 & 24.0 & 192.000 & 61.935 & 120.0 & 120 & 960 \\
\hline Total & 78,000 & 216.5 & 1732.000 & 558.710 & 390.0 & 1,083 & 8,660 \\
\hline Digital angiography & 1,000 & 46.0 & 230.000 & 74.194 & 5.0 & 230 & 1,150 \\
\hline Ultrasound & 6,000 & 240.0 & 62.880 & 29.943 & 30.0 & 1,200 & 314 \\
\hline Nuclear medicine & 2,750 & 16.5 & 2.162 & 1.029 & 13.8 & 83 & 11 \\
\hline Computed tomography & 8,000 & 200.0 & 104.800 & 49.905 & 40.0 & 1,000 & 524 \\
\hline Magnetic resonance & 4,250 & 118.8 & 62.225 & 29.631 & 21.3 & 594 & 311 \\
\hline Digital imaging & 22,000 & 621.3 & 462.067 & 184.701 & 110.0 & 3,106 & 2,310 \\
\hline Total & 100,000 & 837.8 & 2194.067 & 743.411 & 500.0 & 4,189 & 10,970 \\
\hline
\end{tabular}


institution specific. The activity model for a department with a yearly caseload of 100,000 examinations in all modalities can be linearly scaled to any desired multiple.

The following key quantitative factors are used in the model:

1. The digital content of images and exams for each modality as shown in Table 1.

2. All activity based on 100,000 total examinations per year.

3. Distribution of yearly examinations by modality as shown in Table 2.

4. Reversible (nondestructive) compression used for archives.

5. All images archived (other options include archiving only selected images or using higher compression for non-selected images, ie, dual compression).

6. All archived images retrieved (options include partial retrieval).

7. A 5-year file retention after date of last activity (options are a 7-year retention and other periods).

8. Both film images and digital images retrieved for all previous modalities for each new patient visit.

9. Only one day's activity of new and retrieved data is maintained in on-line active files (options include multiple days).

An implementation schedule has been developed as an example of the progressive introduction of PACS at a hypothetical large aggressive institution with substantial human and fiscal resources. The first and second decades of this example are shown on Figures 1 and 2, respectively. The schedule strategy has been developed principally from the user's standpoint based on the qualitative and quantitative guidelines. The rationale for the planning is driven by the interactive factors of the rate of user transition and acceptance, the expected evolution of the hardware, the evolution of the software and systems configurations, and the extension of the boundary of the digital environment to the radiographic subspecialties as there are quality benefits to do so. A primary consideration for the transition from a film-based archival system to PACS in a clinical setting is the accommodation of the changes by the professional and technical staff so that there is confidence in the continuity and quality of the clinical service. It is assumed that the two highly qualified in-house PACS experts will be able to support up to four or sometimes five major schedule subprograms at any one time. Their success in assisting the professional and technical staff in the transitions would be instrumental in assuring the success and timeliness of the program.

As indicated in Figure 1, planning for PACS physical implementation would take place in the first year of the schedule with the first generation components being installed in the second year. Some of the abbreviations used in Figures 1 and 2 are necessarily terse: GEN refers to the generation of equipment; LAN refers to local area network; W/S to work-

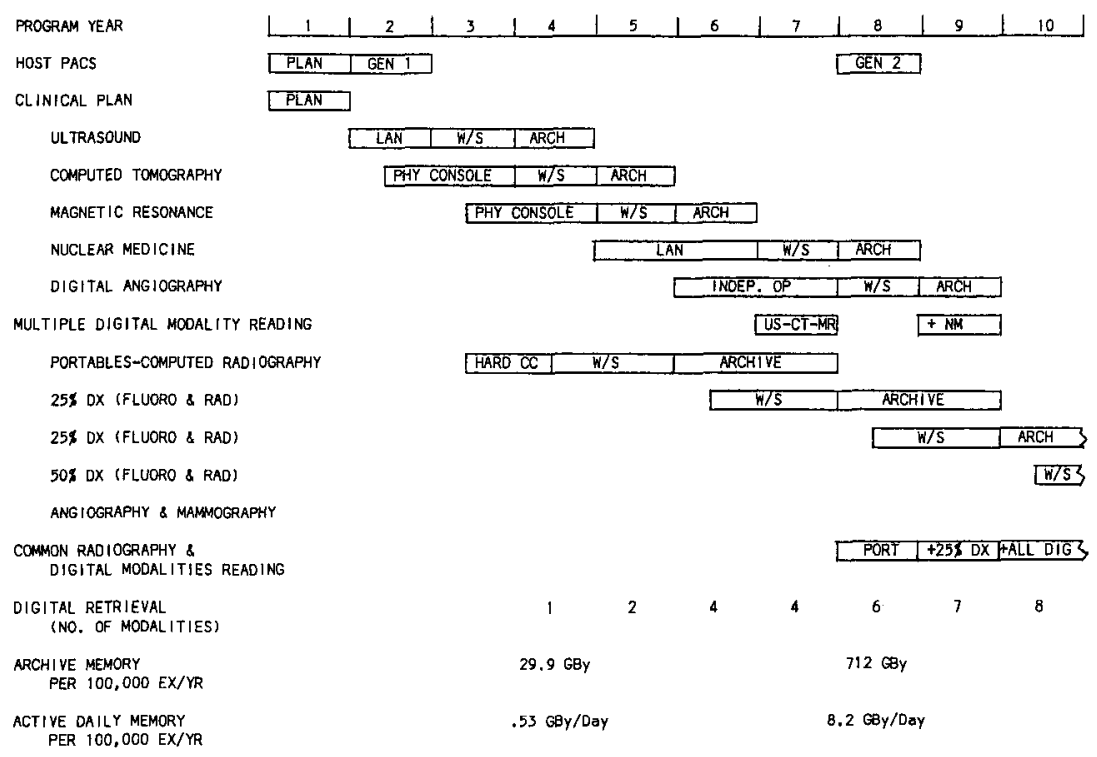

Fig 1. Example of the progressive introduction of PACS in the first decade. 
Fig 2. Example of the progressive introduction of PACS in the second decade.

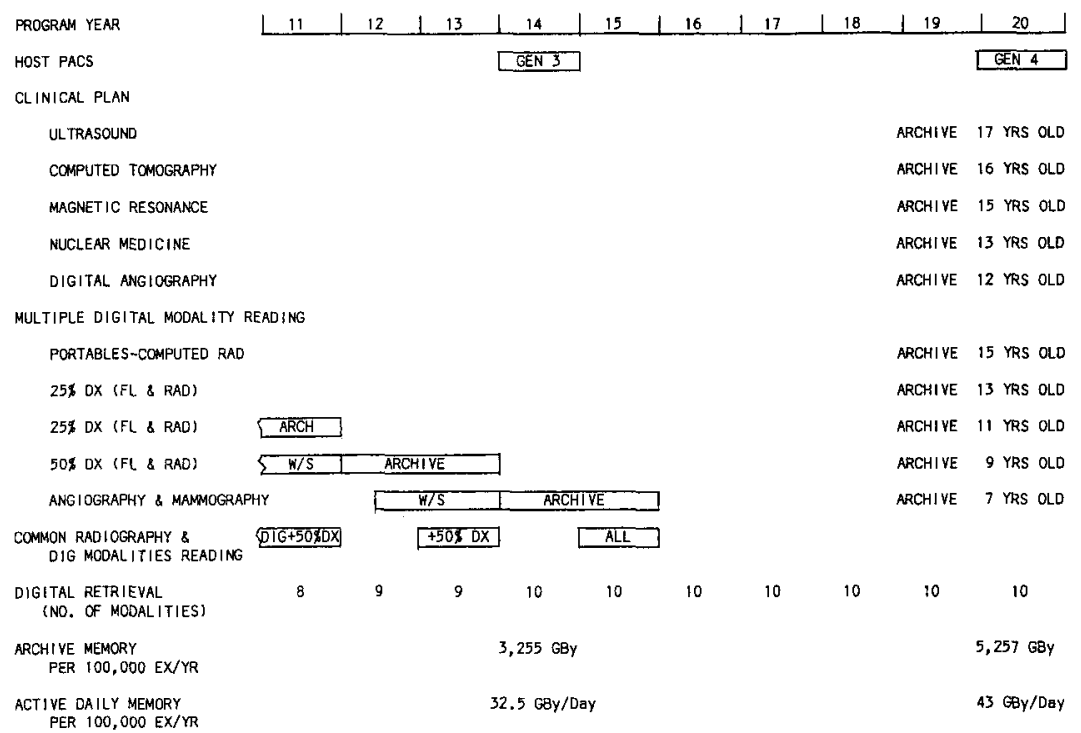

station; ARCH to archived images; INDEP OP to independent operation; HARD CC to hard copy; and DX to diagnostic radiography.

For PACS to fully evolve in the radiology department environment all reading must eventually be done exclusively from soft copy, ie, from workstation images rather than from film. The difficulty and complexity of introducing any one modality into the PACS can be illustrated by considering the example of computed tomography. Currently about $90 \%$ of computed tomography is interpreted from film alone with perhaps $10 \%$ being supplemented by using the physician's console. In the evolution of PACS, a first step would be the transition of all radiologists to read computed tomography using soft copy images on the CT physician's console, supported by hard copy. A next step would be reading from dedicated workstations of a PACS without necessarily archiving the images (still with the film backup), and finally workstation interpretation with images being transferred to and from the PACS archive and with no films being generated or retained. This will require considerable modification to radiologists' practices and also continued technical improvement of workstations with great interactive evolution in workstation software relating to image presentation and navigation.

Soft copy reading for computed tomography is anticipated to begin in the second half of the second year and continue through the third year, followed by extension to workstations and then to use of the PACS archive. The incorporation of ultrasound into PACS is scheduled before computed tomography, and magnetic resonance is programmed to be about 1 year behind computed tomography, with the evolution of other digital modalities following later as shown. Ultrasound was selected for the first introduction because of the expected greater ease of soft copy reading for ultrasound and the longer expected transition cycle for computed tomography.

It is believed that the successful evolution of the general digital environment will be highly dependent on the effective use of computed radiography (sensitized plate technology) to provide diagnostic quality benefits. The introduction of computed radiography for portable radiographic examinations is shown in the third program year, followed by reading from PACS workstations for 18 months and then full archiving and further refinement in the sixth and seventh program years. If computed radiography continues to show diagnostic benefits for an increasing number of applications, the extension of PACS coverage would continue to grow. In what is probably a very aggressive schedule, the growth is shown in 24-month cycles reaching $50 \%$ of the radiographic and fluoroscopic case load in the 10th program year, and culminating with angiography and mammography in the fourteenth and fifteenth program years.

To provide the radiologist with correlative images from prior studies, it will be necessary to 
provide PACS workstations in the reading areas for all modalities as soon as the first modality is archived. The progressive increase in the number of modalities available for retrieval is shown in the lower part of the schedule on Figures 1 and 2.

A key point in the development of the overall PACS is the use of multiple-use workstations to read studies for a multiplicity of modalities. It is anticipated that these workstations, supporting multiple digital modalities, would first be used for the combination of ultrasound, computed tomography, and magnetic resonance just after the last of these modalities is accommodated in the sixth program year. The image presentation, manipulation, and navigation requirements for each of the modalities will need to be provided by the multiple-use workstation. The addition of nuclear medicine reading to the workstations would occur in about the ninth year when nuclear medicine has been incorporated into the archive.

Common workstations, defined as supporting radiography reading as well as digital modality reading, require higher resolution per image than is needed for the digital modality images. The evolution of computed radiography for portable studies suggests common workstations might be in use by the eighth year with the first portion of the routine diagnostic radiographic activity added in the ninth year. The addition of reading for all the digital modalities to these workstations is shown in the tenth year. This will necessitate providing the higher resolution image capability needed for the radiographic studies as well as the extensive image navigation capabilities needed to handle the large number of lower resolution images needed for digital modalities.

\section{QUANTITATIVE REQUIREMENTS}

The schedule provides a chronology for the digital image archive for each modality. Using this information and the dynamic patient visit and film file model, the required size of the cumulative archive and the required amount of daily active memory for new and retrieved images can be calculated. Table 3 indicates the size of the required cumulative digital archive by modality and program year for the first 2 decades. Similarly the amount of active memory needed to handle the data generated and retrieved on a typical day is shown in Table 4. Depending on the PACS architecture, the active memory could be centralized or could be

Table 3. Total Required Size of the Digital Archive (With Compressed Data)

\begin{tabular}{|c|c|c|c|c|c|c|c|c|c|c|c|}
\hline $\begin{array}{c}\text { Program } \\
\text { Year }\end{array}$ & US & CT & MR & NM & $\begin{array}{l}\text { DIG } \\
\text { ANG }\end{array}$ & $\begin{array}{c}\text { PORT } \\
\text { CR }\end{array}$ & $\begin{array}{l}25 \% \\
\text { DX }\end{array}$ & $\begin{array}{l}25 \% \\
D X\end{array}$ & $\begin{array}{c}50 \% \\
\text { DX }\end{array}$ & $\begin{array}{l}\text { ANG/ } \\
\text { MAM }\end{array}$ & $\begin{array}{l}\text { TOTAL } \\
\text { (Gbyte) }\end{array}$ \\
\hline \multicolumn{12}{|l|}{1} \\
\hline \multicolumn{12}{|l|}{2} \\
\hline \multicolumn{12}{|l|}{3} \\
\hline 4 & 30 & & & & & & & & & & 30 \\
\hline 5 & 60 & 50 & & & & & & & & & 110 \\
\hline 6 & 90 & 100 & 30 & & & 62 & & & & & 281 \\
\hline 7 & 120 & 150 & 59 & & & 124 & & & & & 453 \\
\hline 8 & 150 & 200 & 89 & 1.0 & & 186 & 87 & & & & 712 \\
\hline 9 & 168 & 250 & 119 & 2.1 & 74 & 248 & 174 & & & & 1,034 \\
\hline 10 & 183 & 281 & 148 & 3.1 & 148 & 310 & 260 & 87 & & & 1,421 \\
\hline 11 & 195 & 306 & 167 & 4.1 & 223 & 348 & 347 & 174 & & & 1,763 \\
\hline 12 & 205 & 325 & 182 & 5.1 & 297 & 379 & 434 & 260 & 174 & & 2,260 \\
\hline 13 & 212 & 341 & 193 & 5.8 & 371 & 404 & 488 & 347 & 347 & & 2,709 \\
\hline 14 & 217 & 353 & 202 & 6.3 & 417 & 423 & 532 & 434 & 521 & 150 & 3,255 \\
\hline 15 & 221 & 362 & 209 & 6.7 & 454 & 438 & 566 & 488 & 694 & 299 & 3,739 \\
\hline 16 & 225 & 369 & 215 & 7.0 & 484 & 449 & 593 & 532 & 868 & 449 & 4,189 \\
\hline 17 & 227 & 375 & 219 & 7.3 & 507 & 458 & 613 & 566 & 976 & 599 & 4,547 \\
\hline 18 & 229 & 379 & 222 & 7.5 & 524 & 465 & 629 & 593 & 1,063 & 748 & 4,860 \\
\hline 19 & 231 & 382 & 225 & 7.6 & 538 & 470 & 642 & 613 & 1,132 & 842 & 5,082 \\
\hline 20 & 232 & 385 & 227 & 7.7 & 549 & 474 & 651 & 629 & 1,185 & 917 & 5,257 \\
\hline Full & 234 & 391 & 232 & 8.1 & 581 & 485 & 679 & 679 & 1,358 & 1,171 & 5,817 \\
\hline
\end{tabular}

NOTE: Data expressed in gigabytes. 
Table 4. Daily Active Memory Requirement for New and Retrieved Data

\begin{tabular}{|c|c|c|c|c|c|c|c|c|c|c|c|}
\hline $\begin{array}{l}\text { Program } \\
\text { Year }\end{array}$ & us & $C T$ & MR & NM & $\begin{array}{l}\text { DIG } \\
\text { ANG }\end{array}$ & $\begin{array}{l}\text { PORT } \\
\text { CR }\end{array}$ & $\begin{array}{l}25 \% \\
\mathrm{DX}\end{array}$ & $\begin{array}{l}25 \% \\
\text { DX }\end{array}$ & $\begin{array}{l}50 \% \\
D X\end{array}$ & $\begin{array}{l}\text { ANG/ } \\
\text { MAM }\end{array}$ & $\begin{array}{l}\text { TOTAL } \\
\text { (Gbyte) }\end{array}$ \\
\hline \multicolumn{12}{|l|}{1} \\
\hline \multicolumn{12}{|l|}{2} \\
\hline \multicolumn{12}{|l|}{3} \\
\hline 4 & 0.53 & & & & & & & & & & 0.53 \\
\hline 5 & 0.69 & 0.89 & & & & & & & & & 1.58 \\
\hline 6 & 0.82 & 1.15 & 0.53 & & & 1.63 & & & & & 4.13 \\
\hline 7 & 0.93 & 1.37 & 0.68 & & & 2.11 & & & & & 5.09 \\
\hline 8 & 1.02 & 1.55 & 0.81 & 0.02 & & 2.51 & 2.29 & & & & 8.20 \\
\hline 9 & 1.09 & 1.70 & 0.92 & 0.02 & 1.96 & 2.85 & 2.96 & & & & 11.50 \\
\hline 10 & 1.14 & 1.81 & 1.01 & 0.03 & 2.53 & 3.12 & 3.52 & 2.29 & & & 15.45 \\
\hline 11 & 1.18 & 1.90 & 1.08 & 0.03 & 3.01 & 3.32 & 3.99 & 2.96 & & & 17.47 \\
\hline 12 & 1.21 & 1.97 & 1.13 & 0.04 & 3.41 & 3.48 & 4.38 & 3.52 & 4.57 & & 23.71 \\
\hline 13 & 1.24 & 2.02 & 1.17 & 0.04 & 3.74 & 3.61 & 4.66 & 3.99 & 5.92 & & 26.39 \\
\hline 14 & 1.26 & 2.06 & 1.20 & 0.04 & 3.98 & 3.70 & 4.88 & 4.38 & 7.04 & 3.94 & 32.48 \\
\hline 15 & 1.27 & 2.09 & 1.22 & 0.04 & 4.17 & 3.78 & 5.05 & 4.66 & 7.97 & 5.10 & 35.35 \\
\hline 16 & 1.28 & 2.12 & 1.24 & 0.04 & 4.32 & 3.83 & 5.19 & 4.88 & 8.75 & 6.07 & 37.72 \\
\hline 17 & 1.29 & 2.14 & 1.26 & 0.04 & 4.43 & 3.88 & 5.29 & 5.05 & 9.32 & 6.88 & 39.58 \\
\hline 18 & 1.30 & 2.15 & 1.27 & 0.04 & 4.52 & 3.91 & 5.37 & 5.19 & 9.76 & 7.55 & 41.06 \\
\hline 19 & 1.30 & 2.16 & 1.28 & 0.04 & 4.59 & 3.94 & 5.43 & 5.29 & 10.10 & 8.03 & 42.16 \\
\hline 20 & 1.31 & 2.17 & 1.28 & 0.04 & 4.65 & 3.96 & 5.48 & 5.37 & 10.37 & 8.42 & 43.05 \\
\hline Full & 1.31 & 2.19 & 1.30 & 0.05 & 4.81 & 4.01 & 5.62 & 5.62 & 11.24 & 9.70 & 45.85 \\
\hline
\end{tabular}

NOTE: Data expressed in gigabytes.

distributed within the PACS. The archive and daily active memory requirements are for a department with 100,000 examinations per year, 5-year file retention, nondestructive data compression, and progressive library ages for the different modalities. These data could also be used to calculate the communication throughout capabilities required to support the activity.

The cumulative totals from Tables 3 and 4 are also summarized for selected years in the lower portion of the implementation schedule (Figs 1, 2). The required archive grows from 29.9 Gbytes per 100,000 total exams/y in the fourth program year to 5,257 Gbytes in the twentieth program year, while the daily memory requirement grows from 0.53 to 43 Gbytes. These growth rates of 176 and 81 times, respectively, over a 16-year period will hopefully allow the enormous growth

Fig 3. Growth of archival memory for progressive introduction of radiology PACS $\{100,000$ examinations/yr; all images archived).

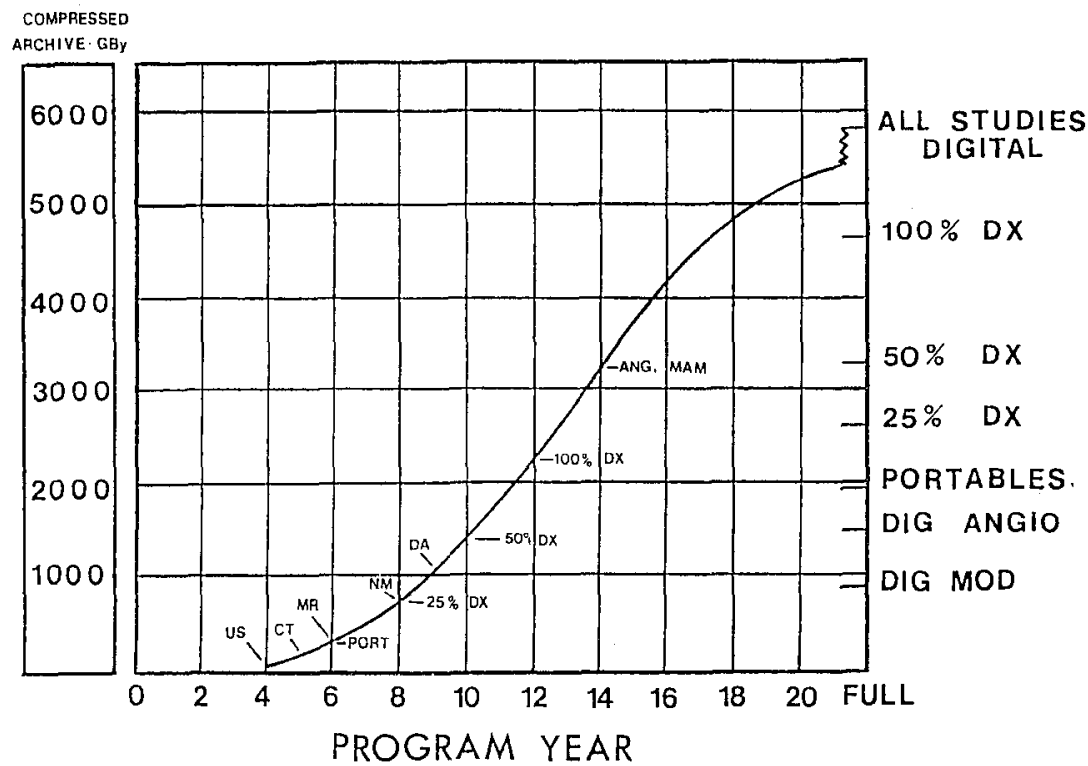




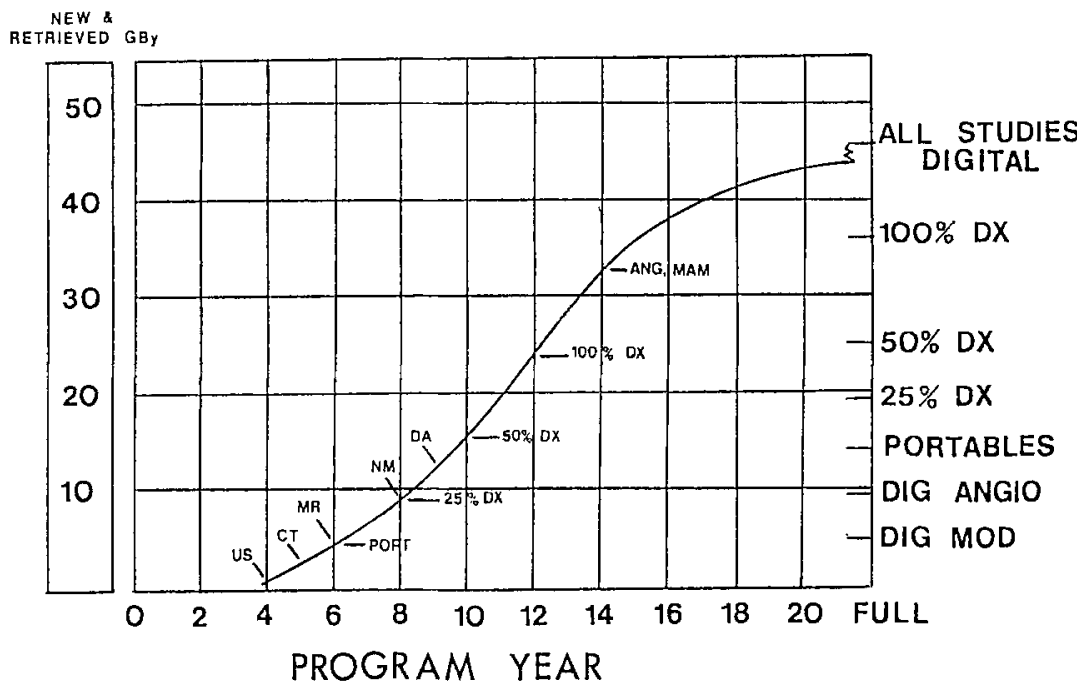

Fig 4. Growth of daily active memory for progressive introduction of radiology PACS $(100,000$ examinations/yr; all images archived). in memory and communication speed requirements to be matched by corresponding improvements in capacity and costs of the component technologies. This growth is shown graphically for the archival storage and daily active memory requirements in Figures 3 and 4, respectively. The growth curves demonstrate the combined effects of the successive introduction of additional modalities and the progressive aging of the archive.

Although the growth of the hardware components comprising the PACS would be relatively continuous during the program, some of the earlier and mid-time components would be expected to become obsolete during the course of the program. If the expected usage cycle for a generation of PACS equipment is about 6 years, (which might be generous in the light of the history of the computer industry), then equipment replacement in about the eighth, fourteenth, and twentieth program years would be expected to provide current technology and to allow capacity expansion with more economic future generations of equipment.

\section{CONCLUSION}

An illustrative strategy and schedule has been provided for the progressive introduction of PACS for a department of radiology. The example suggests that a program timetable of 1.5 to 2 decades might be reasonable for the full implementation of digital technology for interpretation, archiving, and retrieval of all images for all modalities and subspecialties of radiology. The corresponding quantitative projection illustrates the growing magnitude of the digital image management task to very substantial levels. This evolution is shown to be the product of many interactive factors, and the continued enthusiasm for pursuing the goal will probably depend on the perceived desirability of the result. The desirability, or acceptability, of the future general digital environment to the institutional user and to the health care system is expected to depend on the potential benefits, especially in the areas of the quality and cost factors relating to the digital modalities and most especially to computed radiography.

\section{REFERENCES}

1. Naylor AF: On the probable evolution of the digital environment for radiology. S CAR 90: Computer applications to assist radiology. Symposia Foundation, Carlsbad, CA, 1990, pp 332-338
2. Naylor AF: A quantitative projection of digital environment activity for radiology departments. S CAR 90: Computer applications to assist radiology. Symposia Foundation, Carlsbad, CA, 1990, pp 339-349 